\title{
Prognostic impact of advanced lung cancer inflammation index (ALI) on patients with adenocarcinoma of esophagogastric junction (AEG) after radical resection
}

\section{Qianchao Liao}

Shantou University Medical College

\section{Zifeng Yang}

Guangdong Academy of Medical Science

Xu Hu

Affiliated Hospital of Guangdong Medical University

Chengbin Zheng

Jieyang People's Hospital

Huolun Feng

Guangdong Academy of Medical Science

\section{Zhenru Deng}

Guangdong Academy of Medical Science

Jiabin Zheng

Guangdong Academy of Medical Science

Junjiang Wang

Guangdong Academy of Medical Science

\section{Weixian Hu}

Guangdong Academy of Medical Science

\section{Yong Li ( $\nabla$ liyong@gdph.org.cn )}

Guangdong Academy of Medical Science

\section{Research Article}

Keywords: Adenocarcinoma of esophagogastric junction, advanced lung cancer inflammation index, prognosis, overall survival, disease-free survival

Posted Date: November 5th, 2021

DOI: https://doi.org/10.21203/rs.3.rs-1020724/v1 
License: (c) (i) This work is licensed under a Creative Commons Attribution 4.0 International License. Read Full License 


\section{Abstract \\ Background}

To evaluate the prognostic impact of the advanced lung cancer index (ALI) in patients with the adenocarcinoma of esophagogastric junction (AEG) after radical resection.

\section{Methods}

The data of patients with AEG after radical resection at Guangdong Provincial People's Hospital from January 2008 to December 2018 were retrieved. The cutoff value of ALI was determined and the prognostic impact of clinicopathological factors and ALI were analyzed. A nomogram based on the independent prognostic factors for overall survival was then built.

\section{Results}

A total of 147 patients were eligible and based on a cutoff of ALI 43.1, 90 (61.2\%) and 57 (38.8\%) patients were classified in a low- (ALIه43.1) and high-ALI (ALIه43.1) group. Multivariate Cox proportional hazard analysis showed that low-ALI was associated with poor overall survival (OS) (pष0.001, HR 2.541, $95 \% \mathrm{Cl} 1.408-4.410)$ and disease-free survival (DFS) ( $p=0.021, \mathrm{HR} 1.789,95 \% \mathrm{Cl} 1.020-2.674)$. In subgroup analysis, low-ALI was independent predictor for OS ( $p=0.001, \mathrm{HR} 2.628,95 \% \mathrm{Cl} 1.467-4.707)$ in stage III/IVA AEG patients. A nomogram for OS estimation was constructed and the C-index was $0.699(95 \% \mathrm{Cl}$ 0.636-0.762) and the calibration plots showed satisfactory consistency between actual observation and nomogram-predicted OS probabilities. Further, satisfactory predictive accuracy for 1-, 2-, and 3-year OS rates with an area under the curve of $0.736,0.712$ and 0.697 , respectively, was observed.

\section{Conclusions}

ALI was an independent prognostic factor for AEG patients after radical resection, and demonstrated promising ability for risk stratification of AEG, especially in advanced-stage disease.

\section{Introduction}

In recent decades, the incidence of the adenocarcinomas of esophagogastric junction (AEG) has increased worldwide[1-3]. Based on the Siewert classification[4], AEG is defined as a tumor with an epicenter crossing $5 \mathrm{~cm}$ above or below the esophagogastric junction. Despite multimodality treatment strategies, the prognosis of AEG, especially advanced stage AEG, remains poor $[5,6]$.

The TNM classification, related biomarkers and clinicopathological factors are essential indications affecting the prognosis of AEG[7-10]. An improved and individualized prognostic model could further 
help to stratify the risk of these patients and better guide their clinical management.

Recently, increasing evidence on systemic inflammation and immune response illustrated their important roles in tumor progression and impact on patients' survival $[11,12]$. Preoperative laboratory blood tests including CRP, neutrophilic cells, platelets count, albumin levels are reported as proxy for patients' inflammatory status[13-16]. Preoperative inflammatory indices such as the neutrophile/lymphocyte ratio (NLR), platelet/lymphocyte ratio (PLR), Glasgow prognostic score (GPS) had been demonstrated to influence prognosis on different types of tumors[17-20].

Recently, the advanced lung cancer inflammatory index (ALI) was reported as an independent marker of poor outcomes in patient with advanced non-small-cell lung cancer[21]. ALI combines BMI, albumin level and NLR into its evaluation. Compared with NLR which mainly reflects patients' inflammatory status, BMI and serum albumin are suggested as biomarker for nutritional condition[22, 23]. Hence, ALI could have a better prospect as a conventional clinical biomarker for simultaneously evaluating both two aspects. The significance of ALI in nasopharyngeal cancer, large B cell lymphomas and esophageal cancer have been studied [24-26], however to our knowledge, the clinical value of ALI on purely AEG patients had not yet been established.

In this study, we aim to assess the clinical significance of ALI in patients with AEG after radical resection and developed a model for easy clinical implementation to better guide their management. We present the following article in accordance with the STROBE reporting checklist.

\section{Methods}

\section{Patients}

The records of patients with AEG who underwent radical resection at Guangdong Provincial Peoples' Hospital (Guangzhou, China), from January 2008 to December 2018, were assessed. Inclusion criteria included: 1. a pathological diagnosis of adenocarcinoma; 2 . underwent radical surgical resection (R0); 3 . the tumor epicenter was located within $5 \mathrm{~cm}$ above or below the esophagogastric junction and tumor invaded the EG junction; 4. absence of distant metastasis; 5 . had completed clinical and follow-up data. Exclusion criteria were: 1. concomitant malignancy. 2. Clinical or follow-up data incomplete.

Patients' clinicopathologic data were extracted from the hospitals inpatient management system and included age, sex, body mass index (BMI), Siewert classification, T stage, lymph node metastasis, lymphovascular invasion (LVI), perineural invasion (PNI), differentiation, tumor size and ALI. The Siewert subtype classification was defined based on the patient's preoperative gastroscopy, computed tomography, digestive tract radiography and postoperative specimen pathological report. Regular postoperative follow-up was performed. Patients were observed at 3-month intervals for 2 years after surgery, and every 6 months for the 3rd to 5th year by reviewing hospital records, yia outpatient clinics and telephone interviews. Pathological staging was based on the 8th edition of the AJCC TNM classification. 
All patients provided signed informed consent for using their data anonymously for research purposes. The study was performed in accordance with the standards of the Declaration of Helsinki.

\section{Laboratory test}

The patients' weight and height were routinely measured after admission. Blood samples were taken within 1 week of surgery. ALI was calculated using the following formula: $A L I=B M I \times$ serum albumin (Alb: $\mathrm{g} / \mathrm{dL}$ )/ (absolute neutrophil count/ absolute lymphocyte count [NLR]), BMI was calculated using the formula: weight $(\mathrm{kg}) /(\text { height }[\mathrm{m}])^{2}[21]$.

\section{Statistical analyses}

Continuous variables are expressed as the mean \pm SD, and categorical variables were reported as frequencies with percentages. For comparing the correlation between ALI and other clinicopathological factors, the Student's t-test or Mann-Whitney U test was used for continuous variables, whereas the $x 2$ test or Fisher's test was used to compare categorical data. Patients' information about ALI, tumor size and overall survival (OS) time were added to the X-tile software (version 3.6.1)[27] for estimating the optimal cutoff value respectively.

Kaplan-Meier method was used to construct the survival curves and were compared by log-rank test. The Cox proportional hazards method was used to compare the HR for death and recurrence. OS was defined as the time between the date of surgery until death from any reasons or last contact. Disease-free survival (DFS) was defined as the time between the date of surgery until tumor recurrence or death from any reasons or the last contact. The clinicopathological variables with a $\mathrm{p} \otimes 0.10$ in univariate analysis were selected for multivariate analysis by Cox regression models. Independent variables from

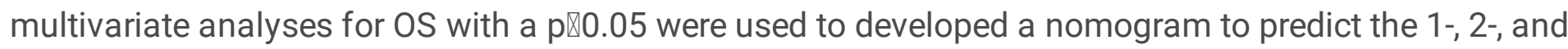
3-year OS survival rates. A C-index value in the range of 0.5-1.0 between predicted and actual outcomes was used to evaluate the predictive ability and discriminative ability of the nomogram model. Calibration plots were used to assess the fitting degree of the nomogram and the relative operating characteristic (ROC) curve with AUC was used to evaluate the discriminative and predictive ability. The statistical analysis was performed using the SPSS (version 22, Chicago, IL, USA), R (www.R-project.org, version 3.6.3) and GraphPad Prism (version 8.2.1) software.

\section{Results}

\section{Study population characteristics}

This study comprised of 147 patients, of whom $102(69.4 \%)$ patients were male. The mean age was $62.54 \pm 9.295$ (range, 30-83) years. Twenty (13.6\%) patients received preoperative treatment while 63 $(42.9 \%)$ had adjuvant therapy. As for anatomic classification, $6(4.1 \%)$ patients were classified as Siewert type I, $73(49.7 \%)$ as Siewert type II, and $68(46.2 \%)$ as Siewert type III. 
For tumor resection, the use of the transabdominal or thoracic approach was determined by preoperative examination findings and surgeons' experience. Sixty (40.8\%) patients underwent the transthoracic approach while 87 (59.2\%) underwent the transabdominal approach. As for pathological factors, 65 (44.2\%) patients had poor differentiation. Perineural invasion and lymphovascular invasion were present in $93(63.3 \%)$ and $59(36.2 \%)$ patients. The cutoff value for tumor size was $3.3 \mathrm{~cm}, 112(76.2 \%)$ patients were in a larger size group. 119 (80.1\%) patients had deeper tumor invasion (pT3-4) and $70(47.6 \%)$ patients had more than 2 positive lymph nodes metastasis (pN2-3).

The determined cutoff value using the X-tile software for ALI was 43.1. Patients with ALI lower than 43.1 were classified into a low-ALI group $(n=90$ [61.2\%]) while those with ALI higher than 43.1 into a high-ALI group $(n=57$ [38.8\%]). The correlation between the clinicopathological factors and ALI is shown in Table 1. More patients with low ALI had larger tumor size $(p=0.011)$. Patients in the high-ALI group had higher preoperative BMI ( $p \otimes 0.001)$, albumin level (pष0.001), and NLR ( $p \otimes 0.001)$. 
Table 1

Table1. Clinicopathological factors for patients with AEG

\begin{tabular}{|c|c|c|c|c|c|}
\hline & & $\mathbf{N}$ & ALI & & $\mathbf{p}$ \\
\hline & & & $\begin{array}{l}\text { Low-ALI } \\
\text { (043.1) }\end{array}$ & $\begin{array}{l}\text { High-ALI }(\geq \\
\text { 43.1) }\end{array}$ & \\
\hline Age & 065 & 85 & 47 & 38 & 0.084 \\
\hline & $\geq 65$ & 62 & 43 & 19 & \\
\hline Sex & Male & 102 & 63 & 39 & 0.840 \\
\hline & Female & 45 & 27 & 18 & \\
\hline Preoperative therapy & No & 127 & 76 & 51 & 0.386 \\
\hline & Yes & 20 & 14 & 6 & \\
\hline postoperative therapy & No & 84 & 46 & 38 & 0.063 \\
\hline & Yes & 63 & 44 & 19 & \\
\hline Siewert classification & $\mathrm{I} / \mathrm{II}$ & 79 & 44 & 35 & 0.138 \\
\hline & III & 68 & 46 & 22 & \\
\hline Surgical Approach & Transthoracic & 60 & 38 & 22 & 0.663 \\
\hline & Transabdominal & 87 & 52 & 35 & \\
\hline Differentiation & poor & 65 & 38 & 27 & 0.540 \\
\hline & moderate/well & 82 & 52 & 30 & \\
\hline Perineural invasion & Absent & 54 & 33 & 21 & 0.983 \\
\hline & Present & 93 & 57 & 36 & \\
\hline Lymphovascular & Absent & 88 & 55 & 33 & 0.698 \\
\hline & Present & 59 & 35 & 24 & \\
\hline Tumor size(cm) & प3.3 & 35 & 15 & 20 & \\
\hline & $\geq 3.3$ & 112 & 75 & 37 & 0.011 \\
\hline Tumor invasion(pT) & pT1-2 & 28 & 13 & 15 & \\
\hline & pT3-4 & 119 & 77 & 42 & 0.074 \\
\hline Lymph nodes & pN0-1 & 77 & 50 & 27 & \\
\hline & pN2-3 & 70 & 40 & 30 & 0.333 \\
\hline
\end{tabular}


Table1. Clinicopathological factors for patients with AEG

\begin{tabular}{|lccc|}
\hline BMI (kg/m2) & 21.24 & $23.57(5.83)$ & $\square 0.001$ \\
\hline Alb (g/L) & $(3.61)$ & $39.40(4.60)$ & $\square 0.001$ \\
\hline NLR & $\begin{array}{l}36.20 \\
(4.28)\end{array}$ & $1.61(0.69)$ & $\square 0.001$ \\
\hline ALI=Advenced lung cancer inflammation index & $2.55(1.03)$ & & \\
\hline BMl:Body mass index;Alb:Albumin;NLR:Neutrophil-lymphocyte ratio & & \\
\hline BMl,Alb and NLR are illustrated as median (IQR) & & \\
\hline
\end{tabular}

\section{Cox regression analyses}

By the time of follow-up, the median follow-up time was 63 month (mean $\pm S D, 68.74 \pm 35.825), 72(49.0 \%)$ patients had died, 53 (58.9\%) in the low-ALI group and 19 (33.3\%) in the high-ALI group. The median OS for the low- and high-ALI group was 43 months and 87 months. The 1, 3, and 5-year OS rate was $84.4 \%$, $58.6 \%$, and $45.8 \%$ respectively for the low-ALI group, while $93.0 \%, 74.7 \%$, and $64.5 \%$ for the high-ALI group (Fig. 1A). The median DFS time was 34 months for the low-ALI group but was not reached for the highALI group. The 1, 3, 5-year DFS rate for low- and high-ALI group was $74.4 \%, 47.1 \%, 43.8 \%$, and $84.2 \%$, $64.3 \%, 54.8 \%$, respectively (Fig. 1B).

Univariate analysis for OS indicated that tumor invasion (pT) ( $p=0.03, \mathrm{HR} 2.261,95 \% \mathrm{Cl} 1.083-4.720)$, lymph node metastasis $(p N)(p=0.008, H R 1.894,95 \% C l 1.185-3.029)$, poor differentiation $(p=0.004, H R$ $1.998,95 \% \mathrm{Cl} 1.253-3.188)$, perineural invasion ( $p=0.002$, $\mathrm{HR} 2.435,95 \% \mathrm{Cl} 1.396-4.246)$, lymphovascular invasion ( $p=0.002$, HR 2.106, 95\% Cl 1.322-3.356), tumor size ( $p=0.003, \mathrm{HR} 2.883,95 \% \mathrm{Cl} 1.421-5.851)$ and low-ALI ( $p=0.005$, HR 2.133, 95\% Cl 1.261-3.605) were associated with poor OS. In multivariate analysis, poor differentiation $(p=0.001, \mathrm{HR} 2.314,95 \% \mathrm{Cl} 1.427-3.752)$, lymphovascular invasion $(p=0.006, \mathrm{HR}$ $1.948,95 \% \mathrm{Cl} 1.209-3.319)$, tumor size ( $p=0.011, \mathrm{HR} 2.543,95 \% \mathrm{Cl} 1.240-5.212)$ and low-ALI ( $p=0.001, \mathrm{HR}$ $2.541,95 \% \mathrm{Cl} 1.408-4.410$ ) were independent risk factors for poor OS. Cox proportional hazard analysis was also performed for predictors of DFS. Low differentiation ( $p=0.005, \mathrm{HR} 1.942,95 \% \mathrm{Cl} 1.224-3.082)$, lymphovascular invasion ( $p=0.009, \mathrm{HR} 1.926,95 \% \mathrm{Cl} 1.175-3.157)$, tumor size $(\mathrm{p}=0.019, \mathrm{HR} 2.259,95 \% \mathrm{Cl}$ 1.140-4.474) and $\mathrm{ALI}(\mathrm{p}=0.021, \mathrm{HR} 1.789,95 \% \mathrm{Cl} 1.092-2.932)$ were identified as independent risk factors for poor DFS (Table 2). 
Table 2

Table2. Multivariate analysis of clinicopathological factors for OS and DFS in patients with AEG

\begin{tabular}{llllll}
\multicolumn{2}{l}{ OS } & & \multicolumn{3}{c}{ DFS } \\
HR & $95 \% \mathrm{Cl}$ & $\mathrm{P}$ & $\mathrm{HR}$ & $\begin{array}{l}95 \% \\
\mathrm{Cl}\end{array}$ & $\mathrm{P}$
\end{tabular}

Differtiation (vs.moderate-well

differentiation)

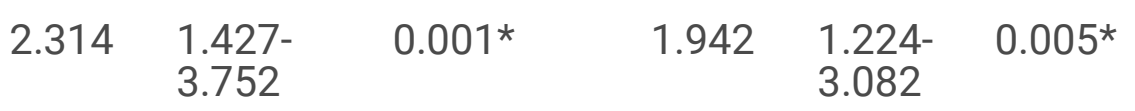

Lymphovascular invasion

(vs.absent)

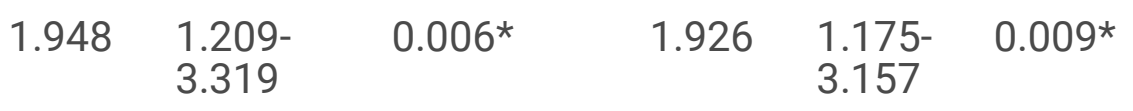

Tumor size (vs.『3.3cm)

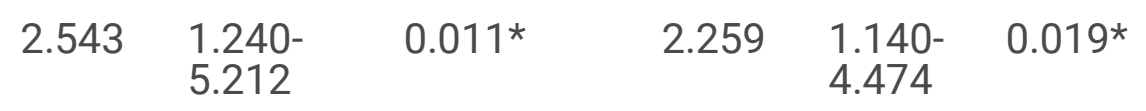

ALI (vs.high-ALI)

$\begin{array}{llllll}2.541 & \begin{array}{l}1.408- \\ 4.410\end{array} & 0.001^{*} & 1.789 & 1.092- & 0.021^{*} \\ 2.932\end{array}$

* $p$ value is statistically significant, $\mathrm{p} \otimes 0.05$

\section{Construction of the nomogram for OS}

Poor differentiation, lymphovascular invasion, tumor size, and low-ALI were the independent prognostic factors for OS used to construct the nomogram (Fig. 2). The C-index for the OS predicting nomogram of patients with AEG was $0.699(95 \% \mathrm{Cl} 0.636-0.762)$. The dotted line in the calibration plot represented the predictive value of the nomogram, while the colorful line represented the actual 1-, 2- and 3-year OS rates. The more cohesive the colorful line to the dotted line, the more precise the nomogram's predictive capability. As shown in Fig. 3A, the colorful lines fluctuated above and below the dotted line, indicating a reliable predictive value of the nomogram. Besides, the AUC for the 1-, 2-, and 3-year OS were $0.736,0.712$, and 0.697 respectively, indicating acceptable discrimination of this nomogram (Fig. 3B).

\section{Subgroup survival analyses of ALI in different stage patients}

Next, we evaluated whether ALI could be a predictor for OS or DFS in different stage AEG. We found that ALI is also independent prognostic predictor for stage III/IVA AEG patients. For these patients, the median OS time was 33 months in the low-ALI group and 87 months in the high-ALI group. 1,3,5-year overall survival rate was $81.4 \%, 47.7 \%$ and $31.0 \%$ respectively for low-ALI group, while $92.3 \%, 68.6 \%$ and $58.1 \%$ for high-ALI group (Fig. 1C). The median disease-free survival time was 27 months in the low-ALI group 
and 45 months in the high-ALI group. 1,3,5-year disease-free survival rate was $71.2 \%, 35.3 \%, 33.2 \%$ for low-ALI group and 79.5\%, 53.5\%, 44.1\% for high-ALI group (Fig. 1D).

In univariate analysis, ALI was statistically significant in stage III/IVA but not Stage I or II for OS $(p=0.005$, HR 2.303, 95\% Cl 1.291-4.108), the p-value for Stage III/IVA did not reach statistical significance for DFS $(p=0.086, H R 1.586,95 \% \mathrm{Cl} 0.086)$. Cox proportional hazard analysis showed that ALI was an independent predictor for OS ( $p=0.001$, HR $2.628,95 \% \mathrm{Cl} 1.291-4.108)$ but not DFS $(p=0.093, \mathrm{HR} 1.589,95 \% \mathrm{Cl} 0.944-$ 2.823) in stage III/IVA patients (Table 3 ).

Table 3

Table3. Multivariate analysis of clinicopathological factors for OS and DFS in stage III/IVA patients

\begin{tabular}{|c|c|c|c|c|c|c|}
\hline & \multicolumn{3}{|c|}{ OS } & \multicolumn{3}{|c|}{ DFS } \\
\hline & HR & $95 \% \mathrm{Cl}$ & $\mathbf{P}$ & HR & $\begin{array}{l}95 \% \\
\mathrm{Cl}\end{array}$ & $\mathbf{P}$ \\
\hline \multicolumn{7}{|l|}{$\begin{array}{l}\text { Differtiation (vs.moderate-well } \\
\text { differentiation) }\end{array}$} \\
\hline & 1.801 & $\begin{array}{l}1.051- \\
3.087\end{array}$ & $0.032 *$ & 1.570 & $\begin{array}{l}0.935- \\
2.636\end{array}$ & 0.088 \\
\hline Perineural invasion(vs.absent) & 2.169 & $\begin{array}{l}1.048- \\
4.489\end{array}$ & $0.037 *$ & 2.360 & $\begin{array}{l}1.135- \\
4.908\end{array}$ & $0.022^{*}$ \\
\hline \multicolumn{7}{|l|}{$\begin{array}{l}\text { Lymphovascular invasion } \\
\text { (vs.absent) }\end{array}$} \\
\hline & 1.350 & $\begin{array}{l}0.730- \\
2.495\end{array}$ & 0.339 & 1.525 & $\begin{array}{l}0.850- \\
2.735\end{array}$ & $0.157 *$ \\
\hline \multicolumn{7}{|l|}{ Tumor size (vs.凶3.3cm) } \\
\hline & 1.846 & $\begin{array}{l}0.713- \\
4.783\end{array}$ & 0.207 & 2.312 & $\begin{array}{l}0.898- \\
5.952\end{array}$ & 0.082 \\
\hline \multicolumn{7}{|l|}{ ALI (vs.high-ALI) } \\
\hline & 2.628 & $\begin{array}{l}1.467- \\
4.707\end{array}$ & $0.001^{*}$ & 1.589 & $\begin{array}{l}0.944- \\
2.823\end{array}$ & 0.093 \\
\hline
\end{tabular}

\section{Discussion}

In recent years, there was rising incidence of $A E G$ in western countries and similar trend also found in East Asia, probably for the sake of obesity, gastroesophageal reflux disease and eradication of Helicobacter pylori $[1,28,29]$. AEG had demonstrated different biological behavior and pathological features compared with either gastric or esophageal cancer[30]. It has attracted more attention and has gradually become a distinct tumor category from esophageal and gastric cancer. Thus, we believed deeper understanding of AEG could benefit to better management of these patients. 
There was accumulating evidence indicating that inflammatory response and nutritional status playing important role in malignancies[11, 12, 31-34]. Several studies had evaluated the inflammatory indexes, including NLR, PLR, GPS, as prognostic predictors in upper gastrointestinal cancers $[35,36]$. In another hand, poor nutritional status, for example, decrease of preoperative albumin level or low BMI value, had demonstrated as indicators for poor prognosis [22, 23,37]. According to previous study, we thought ALI could be a potential surrogate combining both two dimensions. ALI's prognostic impact had been evaluated in several cancers $[21,24,26]$ and it also had been showed to be independent predictor in gastric and esophageal cancer. However, in Yin's et al [38] study tumor mostly located at gastric body or pylorus and Tan's et al [25] study mostly included squamous cell carcinoma of esophagus. Since then, the prgnostic value of ALI for patients with AEG remain unclear.

The major finding of this study is the promising prognostic impact of ALI in patients with AEG after radical resection. Compared with the high-ALI group, patients in the low-ALI group had a worse prognosis. Multivariate analyses showed that ALI was an independent risk factor for both OS and DFS. In line with the previous studies in several other malignancies, our study indicated ALI as a feasible predictor for both OS and DFS in patients with AEG after radical resection. Even though several nomogram models had been used for the prediction of prognosis of $\operatorname{AEG}[39,40]$, this is the first to incorporate ALI into the survival model of AEG patients after radical resection. The $\mathrm{C}$-index of our propoesd nomogram was 0.699 , showing acceptable discrimninatory ability.

In this study, besides ALI, poor differentiation, lymphovascular invasion and tumor size were also recognized as independent prognostic factors for AEG. Poor differentiation already been incorporated into the AJCC classification for esophageal cancer as histologic Grade 3. Poor differentiation was also found to be a predictor for deeper tumor invasion and lymph nodes metastasis in gastric cancer[41, 42]. Even though not well established in AEG, we believed that the prognostic value of differentiation status deserves more investigation. Lymphovascular invasion, the presence of malignant cell within endotheliallined space, is correlated with the ability of the cancer to metastasize[43]. In line with a previous study, it is also considered as a prognostic factor for AEG[44]. Tumor size had been used as a staging method in several solid tumors like lung and breast cancer. Enlarged tumor mass was correlated with increased risk of other adverse pathological features[45]. In our study, more patients in low-ALI group had enlarged tumor size $(\geq 3.3)(p=0.003)$. This might be related to a larger tumor mass inducing more intense inflammatory response and consuming more host nutrition. Moreover, as tumor size increased, it might invade beyond the esophagogastric junction, leading to an increased risk of mediastinal metastasis, a unique feature for patients with AEG.

Another important finding of this study was the prognostic impact of ALI in patients with stage III/IVA AEG. Since Siewert type I and II patients are staged as esophageal cancer, tumor with T4aN2, T4bNO-2 and any $T$ stage with N3 would be staged as stage IVA. Regarding locally advanced disease without distant metastasis, we combined stage IVA patients with stage III patients in the same subgroup for survival analysis. Up to now, the optimal management strategy for AEG is still under debate. Treatment methods had involved preoperative chemoradiotherapy, perioperative chemotherapy or postoperative 
chemotherapy regimens based on different RCT results [46-48]. Although multimodality therapy had improved survival outcomes compared to surgery alone, the life expectancy of AEG patients, even after radical resection, remains poor, especially in patients with advanced disease. In East Asia, the ACTS-GC study demonstrated that 1-year of S-1 administration was associated with a 5 -year OS rates of $50.2 \%$ and 5 -year RFS rates of $37.6 \%$ in stage IIIB gastric cancer patients, which was worse than stage II or IIIA patients (5-year OS rates, II vs IIIA, $84.2 \%$ vs $67.1 \%$, and 5 -year RFS rates, II vs IIIA, $79.2 \%$ vs $61.4 \%$, respectively) [47]. Our study demonstrated that low-ALI group patients with stage III/IVA had a worse prognosis. The 5-year DFS rates were $44.1 \%$ in the high-ALI group and $33.2 \%$ in the low-ALI group. The 5year OS rates were $58.1 \%$ and $31.0 \%$ in the high-ALI group and the low-ALI group. This suggested that stage III/ IVA AEG patients with low ALI levels might have a greater chance of suffering from tumor relapse and death from any cause. We, therefore, suggest closer follow-up may be needed for patients with low-ALI. Moreover, the interim analysis of the JACCRO GC-07 study indicated that S-1 plus docetaxel had superior oncological outcome than S-1 single agent for stage III gastric cancer [49], suggesting that advanced stage patients could further benefit from the more intensive regimen. Whether the difference in ALI level would lead to different treatment response and help for regimen selection or dose/course adjustment needs further investigation.

Despite the promising findings of this study, there were several limitations worth mentioning. First, this was a retrospective study from a single medical institution and the sample size was relatively small. Second, the distribution of cases was uneven, i.e. relatively fewer number stage I patients, which may have affected the results obtained, to some extent. To overcome these limitations, a multicenter prospective study might be needed to clarify the clinical value of ALI in patients with AEG.

\section{Conclusion}

In conclusion, this study identified preoperative ALI as prognostic marker for OS and DFS in patients with AEG after radical resection. Advanced stage AEG patients with low-ALI had worse oncological outcome and assessment of ALI may help for management strategy adjustment.

\section{Abbreviations}

\section{ALI}

Advanced lung cancer inflammatory index

AEG

Adenocarcinoma of esophagogastric junction

OS

Overall survival

DFS

Disease-free survival

NLR

Neutrophile/lymphocyte ratio 
PLR

Platelet/lymphocyte ratio

GPS

Glasgow prognostic score

BMI

body mass index

LVI

lymphovascular invasion

$\mathrm{PNI}$

perineural invasion

\section{Declarations}

\section{Ethical approval and consent to participate}

The study was approved by both the research ethics committee of Guangdong Provincial People's Hospital and Guangdong Provincial Hospital of Chinese Medicine and was performed in accordance with the standards of the Declaration of Helsinki. Patients admitted to the hospital have signed informed consent forms stating that clinical data during hospitalization can be used for anonymous retrospective studies, and it has been approved by the hospital ethics committee.

\section{Consent for publication}

Written informed consent for publication was obtained from all participants.

\section{Availability of data and materials}

The datasets used or analysed during the current study are available from the corresponding author on reasonable request.

\section{Competing interests}

The authors declare that they have no competing interests.

\section{Funding}

Project supported by the Science and Technology Planning Project of Guangdong Province of China (No. 2019A050510025).

\section{Authors' contributions}

Guarantor of the article:LY. Conceptualization: LY and WJJ; Study design: LQC and YZF; Acquisition of data: HX, ZCB, DZR, FHL; Methodology: LQC and YZF; Formal analysis and interpretation: LQC, YZF; 
Writing-original draft preparation: LQC and YZF; Writing-review and editing: LY and WJJ; Statistical analysis: LQC, ZJB. Study supervision: LY and HWX. All authors read and approved the final manuscript.

\section{Acknowledgements}

Not applicable

\section{References}

1. Liu K, Yang K, Zhang W et al. Changes of Esophagogastric Junctional Adenocarcinoma and Gastroesophageal Reflux Disease Among Surgical Patients During 1988-2012: A Single-institution, High-volume Experience in China. Annals of surgery 263(1), 88-95 (2016).

2. Matsuno K, Ishihara R, Ohmori M et al. Time trends in the incidence of esophageal adenocarcinoma, gastric adenocarcinoma, and superficial esophagogastric junction adenocarcinoma. Journal of gastroenterology 54(9), 784-791 (2019).

3. Steevens J, Botterweck AA, Dirx MJ, Van Den Brandt PA, Schouten LJ. Trends in incidence of oesophageal and stomach cancer subtypes in Europe. European journal of gastroenterology \& hepatology 22(6), 669-678 (2010).

4. Siewert JR. Adenocarcinoma of the esophago-gastric junction. Gastric cancer: official journal of the International Gastric Cancer Association and the Japanese Gastric Cancer Association 2(2), 87-88 (1999).

5. Deng JY, Liang H. Adenocarcinoma of esophagogastric junction. Chinese journal of cancer research = Chung-kuo yen cheng yen chiu 26(4), 362-363 (2014).

6. Hosoda K, Yamashita K, Katada N, Watanabe M. Overview of multimodal therapy for adenocarcinoma of the esophagogastric junction. General thoracic and cardiovascular surgery 63(10), 549-556 (2015).

7. Hosokawa Y, Kinoshita T, Konishi M et al. Clinicopathological features and prognostic factors of adenocarcinoma of the esophagogastric junction according to Siewert classification: experiences at a single institution in Japan. Annals of surgical oncology 19(2), 677-683 (2012).

8. Lagarde SM, Ten Kate FJ, Richel DJ, Offerhaus GJ, Van Lanschot JJ. Molecular prognostic factors in adenocarcinoma of the esophagus and gastroesophageal junction. Annals of surgical oncology 14(2), 977-991 (2007).

9. Li Y, Wang M, Zhu X, Cao X, Wu Y, Fang F. Prognostic Significance of CIP2A in Esophagogastric Junction Adenocarcinoma: A Study of 65 Patients and a Meta-Analysis. Disease markers 2019 2312439 (2019).

10. Von Rahden BH, Stein HJ, Feith M, Becker K, Siewert JR. Lymphatic vessel invasion as a prognostic factor in patients with primary resected adenocarcinomas of the esophagogastric junction. Journal of clinical oncology: official journal of the American Society of Clinical Oncology 23(4), 874-879 (2005). 
11. Colotta F, Allavena P, Sica A, Garlanda C, Mantovani A. Cancer-related inflammation, the seventh hallmark of cancer: links to genetic instability. Carcinogenesis 30(7), 1073-1081 (2009).

12. Shinko D, Diakos Cl, Clarke SJ, Charles KA. Cancer-Related Systemic Inflammation: The Challenges and Therapeutic Opportunities for Personalized Medicine. Clinical pharmacology and therapeutics 102(4), 599-610 (2017).

13. Arroyo V, García-Martinez R, Salvatella X. Human serum albumin, systemic inflammation, and cirrhosis. Journal of hepatology 61(2), 396-407 (2014).

14. Kolaczkowska E, Kubes P. Neutrophil recruitment and function in health and inflammation. Nature reviews. Immunology 13(3), 159-175 (2013).

15. Margraf A, Zarbock A. Platelets in Inflammation and Resolution. Journal of immunology (Baltimore, Md.: 1950) 203(9), 2357-2367 (2019).

16. Pepys MB, Baltz ML. Acute phase proteins with special reference to C-reactive protein and related proteins (pentaxins) and serum amyloid A protein. Advances in immunology 34 141-212 (1983).

17. Cheng CB, Zhang QX, Zhuang LP, Sun JW. Prognostic value of lymphocyte-to-C-reactive protein ratio in patients with gastric cancer after surgery: a multicentre study. Japanese journal of clinical oncology 50(10), 1141-1149 (2020).

18. Huang $\mathrm{X}, \mathrm{Hu} \mathrm{H}$, Zhang $\mathrm{W}$, Shao Z. Prognostic value of prognostic nutritional index and systemic immune-inflammation index in patients with osteosarcoma. Journal of cellular physiology 234(10), 18408-18414 (2019).

19. Mcmillan DC. The systemic inflammation-based Glasgow Prognostic Score: a decade of experience in patients with cancer. Cancer treatment reviews 39(5), 534-540 (2013).

20. Song $Y$, Yang $Y$, Gao $P$ et al. The preoperative neutrophil to lymphocyte ratio is a superior indicator of prognosis compared with other inflammatory biomarkers in resectable colorectal cancer. BMC cancer 17(1), 744 (2017).

21. Jafri SH, Shi R, Mills G. Advance lung cancer inflammation index (ALI) at diagnosis is a prognostic marker in patients with metastatic non-small cell lung cancer (NSCLC): a retrospective review. BMC cancer13 158 (2013).

22. Crumley $A B$, Stuart RC, Mckernan $M$, Mcmillan DC. Is hypoalbuminemia an independent prognostic factor in patients with gastric cancer? World journal of surgery 34(10), 2393-2398 (2010).

23. Feng $F$, Zheng G, Guo $X$ et al. Impact of body mass index on surgical outcomes of gastric cancer. BMC cancer 18(1), 151 (2018).

24. Park YH, Yi HG, Lee MH, Kim CS, Lim JH. Prognostic Value of the Pretreatment Advanced Lung Cancer Inflammation Index (ALI) in Diffuse Large B Cell Lymphoma Patients Treated with R-CHOP Chemotherapy. Acta haematologica 137(2), 76-85 (2017).

25. Tan X, Peng H, Gu P, Chen M, Wang Y. Prognostic Significance of the L3 Skeletal Muscle Index and Advanced Lung Cancer Inflammation Index in Elderly Patients with Esophageal Cancer. Cancer management and research 13 3133-3143 (2021). 
26. Topkan E, Ozdemir Y, Kucuk A et al. Low Advanced Lung Cancer Inflammation Index Predicts Poor Prognosis in Locally Advanced Nasopharyngeal Carcinoma Patients Treated with Definitive Concurrent Chemoradiotherapy. Journal of oncology 20203127275 (2020).

27. Camp RL, Dolled-Filhart M, Rimm DL. X-tile: a new bio-informatics tool for biomarker assessment and outcome-based cut-point optimization. Clinical cancer research: an official journal of the American Association for Cancer Research 10(21), 7252-7259 (2004).

28. Colquhoun A, Arnold M, Ferlay J, Goodman KJ, Forman D, Soerjomataram I. Global patterns of cardia and non-cardia gastric cancer incidence in 2012. Gut 64(12), 1881-1888 (2015).

29. Kusano C, Gotoda T, Khor CJ et al. Changing trends in the proportion of adenocarcinoma of the esophagogastric junction in a large tertiary referral center in Japan. Journal of gastroenterology and hepatology 23(11), 1662-1665 (2008).

30. Feith M, Stein HJ, Siewert JR. Adenocarcinoma of the esophagogastric junction: surgical therapy based on 1602 consecutive resected patients. Surgical oncology clinics of North America 15(4), 751-764 (2006).

31. Diakos Cl, Charles KA, Mcmillan DC, Clarke SJ. Cancer-related inflammation and treatment effectiveness. The Lancet. Oncology 15(11), e493-503 (2014).

32. Galdiero MR, Marone G, Mantovani A. Cancer Inflammation and Cytokines. Cold Spring Harbor perspectives in biology 10(8), (2018).

33. Mantzorou M, Koutelidakis A, Theocharis S, Giaginis C. Clinical Value of Nutritional Status in Cancer: What is its Impact and how it Affects Disease Progression and Prognosis? Nutrition and cancer 69(8), 1151-1176 (2017).

34. Na BG, Han SS, Cho YA et al. Nutritional Status of Patients with Cancer: A Prospective Cohort Study of 1,588 Hospitalized Patients. Nutrition and cancer 70(8), 1228-1236 (2018).

35. Powell A, Eley C, Chin C, Coxon AH, Christian A, Lewis WG. Prognostic significance of serum inflammatory markers in esophageal cancer. Esophagus: official journal of the Japan Esophageal Society 18(2), 267-277 (2021).

36. Powell A, Parkinson D, Patel N, Chan D, Christian A, Lewis WG. Prognostic Significance of Serum Inflammatory Markers in Gastric Cancer. Journal of gastrointestinal surgery: official journal of the Society for Surgery of the Alimentary Tract 22(4), 595-605 (2018).

37. Wada T, Kunisaki C, Ono HA, Makino H, Akiyama H, Endo I. Implications of BMI for the Prognosis of Gastric Cancer among the Japanese Population. Digestive surgery 32(6), 480-486 (2015).

38. Yin $C$, Toiyama $Y$, Okugawa $Y$ et al. Clinical significance of advanced lung cancer inflammation index, a nutritional and inflammation index, in gastric cancer patients after surgical resection: $A$ propensity score matching analysis. Clinical nutrition (Edinburgh, Scotland) 40(3), 1130-1136 (2021).

39. Chen K, Deng X, Yang Z et al. Survival nomogram for patients with metastatic siewert type II adenocarcinoma of the esophagogastric junction: a population-based study. Expert review of gastroenterology \& hepatology 14(8), 757-764 (2020). 
40. Liu F, Zhou R, Jiang F, Liu G, Li K, Zhu G. Proposal of a Nomogram for Predicting Survival in Patients with Siewert Type II Adenocarcinoma of the Esophagogastric Junction After Preoperative Radiation. Annals of surgical oncology 26(5), 1292-1300 (2019).

41. Chen JN, Wang QW, Zhang QW, Tang ZR, Li XB. Poorly differentiated is more significant than signet ring cell component for lymph node metastasis in mixed-type early gastric cancer: a retrospective study from a large-volume hospital. Surgical endoscopy 35(4), 1558-1565 (2021).

42. Lee SM, Yang S, Joo M et al. Poorly differentiated component in gastric pinch biopsies predicts submucosal invasion. Diagnostic pathology 934 (2014).

43. Li ZG, Zhu H, Shi H et al. Lymphovascular invasion and nodal metastasis in intramucosal adenocarcinoma of the esophagus and esophagogastric junction. Journal of digestive diseases 16(4), 197-204 (2015).

44. Zheng C, Feng X, Zheng J et al. Lymphovascular Invasion as a Prognostic Factor in Non-Metastatic Adenocarcinoma of Esophagogastric Junction After Radical Surgery. Cancer management and research 12 12791-12799 (2020).

45. Hoshino I, Gunji H, Ishige F et al. Surgical treatment strategy for esophagogastric junction cancers based on the tumor diameter. BMC surgery 19(1), 152 (2019).

46. Al-Batran SE, Homann N, Pauligk C et al. Perioperative chemotherapy with fluorouracil plus leucovorin, oxaliplatin, and docetaxel versus fluorouracil or capecitabine plus cisplatin and epirubicin for locally advanced, resectable gastric or gastro-oesophageal junction adenocarcinoma (FLOT4): a randomised, phase 2/3 trial. Lancet (London, England) 393(10184), 1948-1957 (2019).

47. Sasako M, Sakuramoto S, Katai H et al. Five-year outcomes of a randomized phase III trial comparing adjuvant chemotherapy with S-1 versus surgery alone in stage II or III gastric cancer. Journal of clinical oncology: official journal of the American Society of Clinical Oncology 29(33), 4387-4393 (2011).

48. Shapiro J, Van Lanschot JJB, Hulshof M et al. Neoadjuvant chemoradiotherapy plus surgery versus surgery alone for oesophageal or junctional cancer (CROSS): Iong-term results of a randomised controlled trial. The Lancet. Oncology 16(9), 1090-1098 (2015).

49. Yoshida K, Kodera Y, Kochi M et al. Addition of Docetaxel to Oral Fluoropyrimidine Improves Efficacy in Patients With Stage III Gastric Cancer: Interim Analysis of JACCRO GC-07, a Randomized Controlled Trial. Journal of clinical oncology: official journal of the American Society of Clinical Oncology 37(15), 1296-1304 (2019).

\section{Figures}



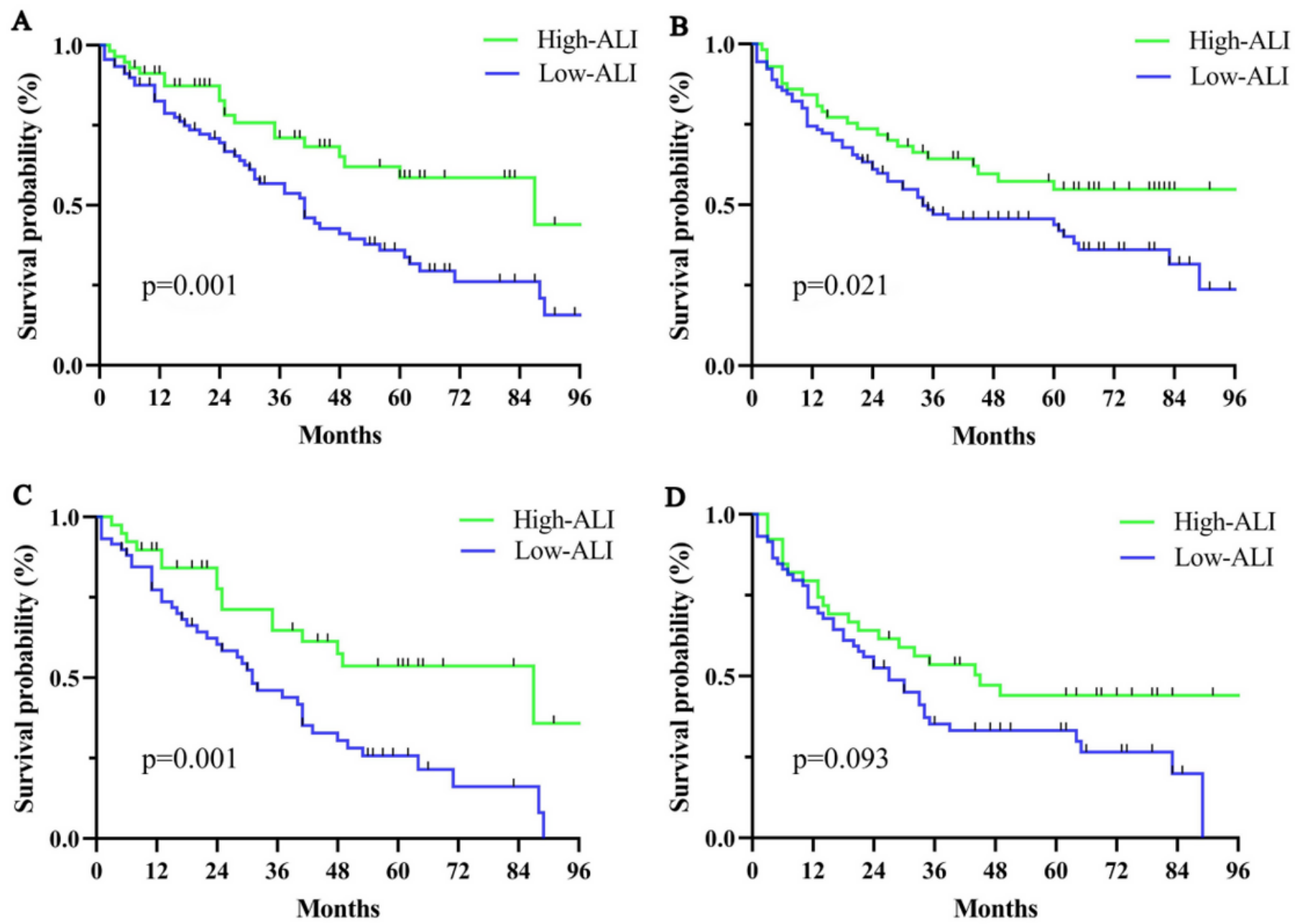

Figure 1

Survival curve for patients with AEG. 1A. Overall survival of patients with AEG. 1B. Disease-free survival of patients with AEG. 1C. Overall survival of patients with stage III/IVA AEG. 1D. Disease-free survival of patients with stage III/IVA AEG. The $x$-axis represents the survival time and $y$-axis represents the survival rate 
Points

$\begin{array}{lllllllllll}0 & 10 & 20 & 30 & 40 & 50 & 60 & 70 & 80 & 90 & 100\end{array}$

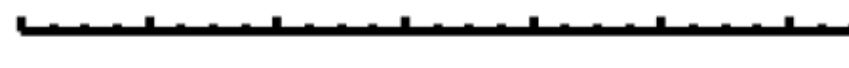

Lymphovascular invasion $\underset{\text { absent }}{\text { present }}$

Differentiation

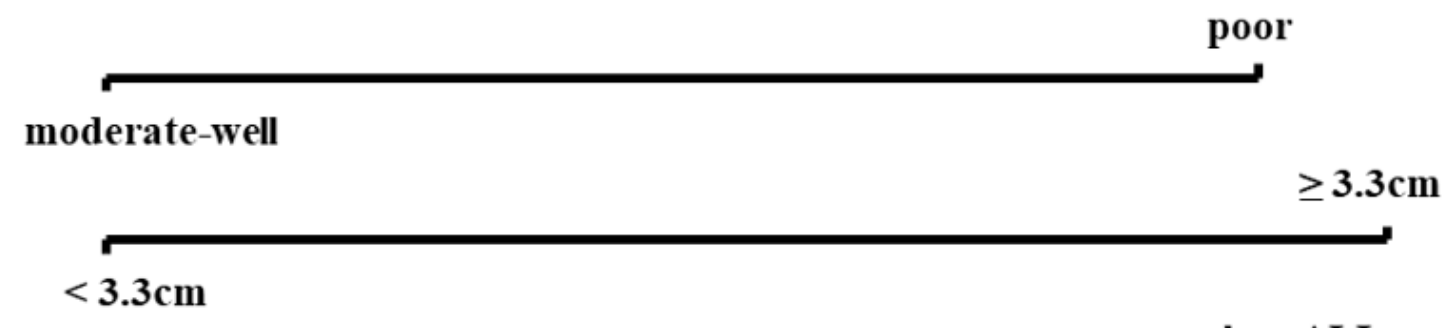

ALI

high-ALI

low-ALI

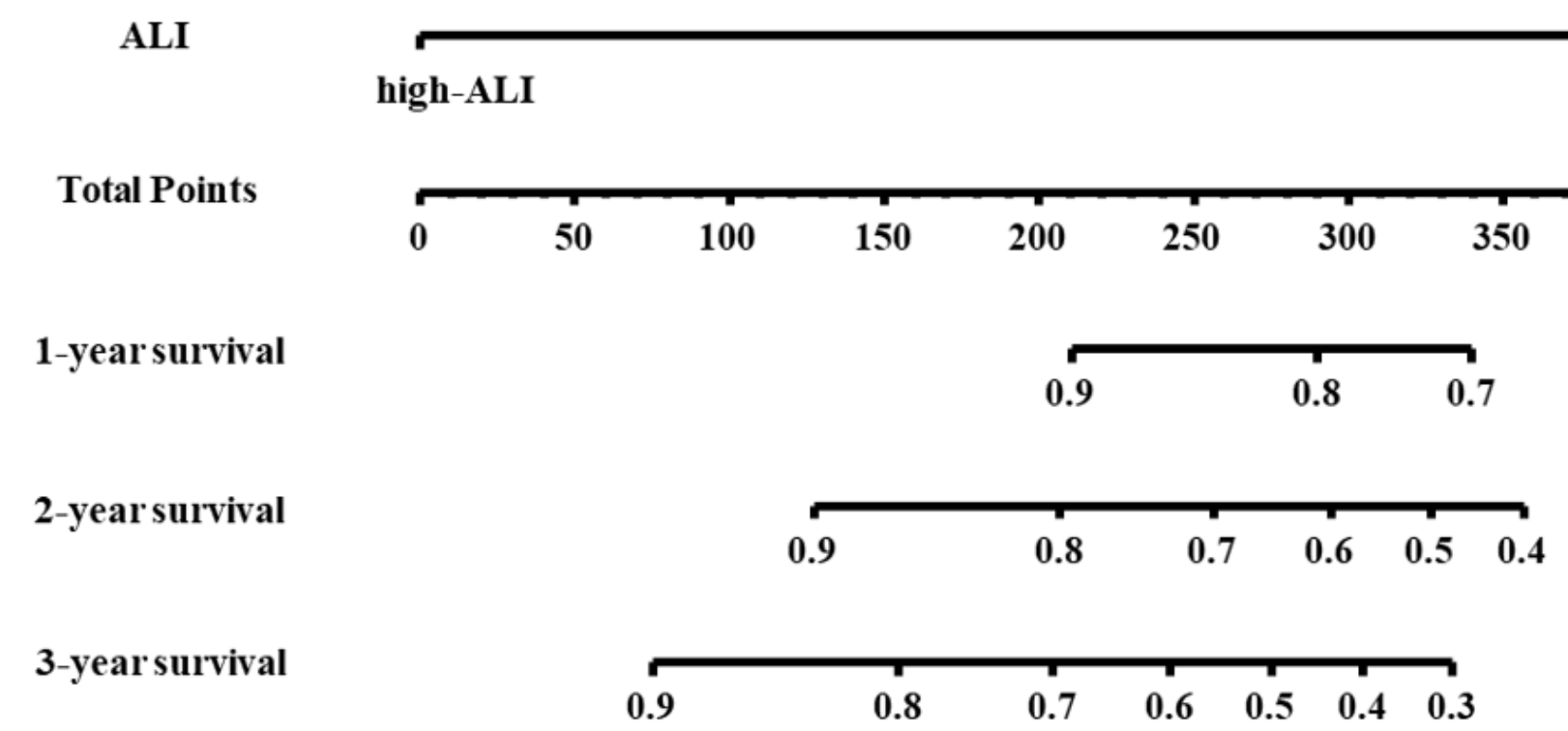

Figure 2

Nomogram to predict the overall survival of AEG. The C-index of this nomogram was $0.699(95 \% \mathrm{Cl}$, 0.636-0.762). The nomogram is constructed based on multivariate analysis for OS for the whole study population. Lymphovascular invasion, poor differentiation, tumor size and ALI are identified as independent prognostic indicators, and these factors show combined effect on OS prediction. 


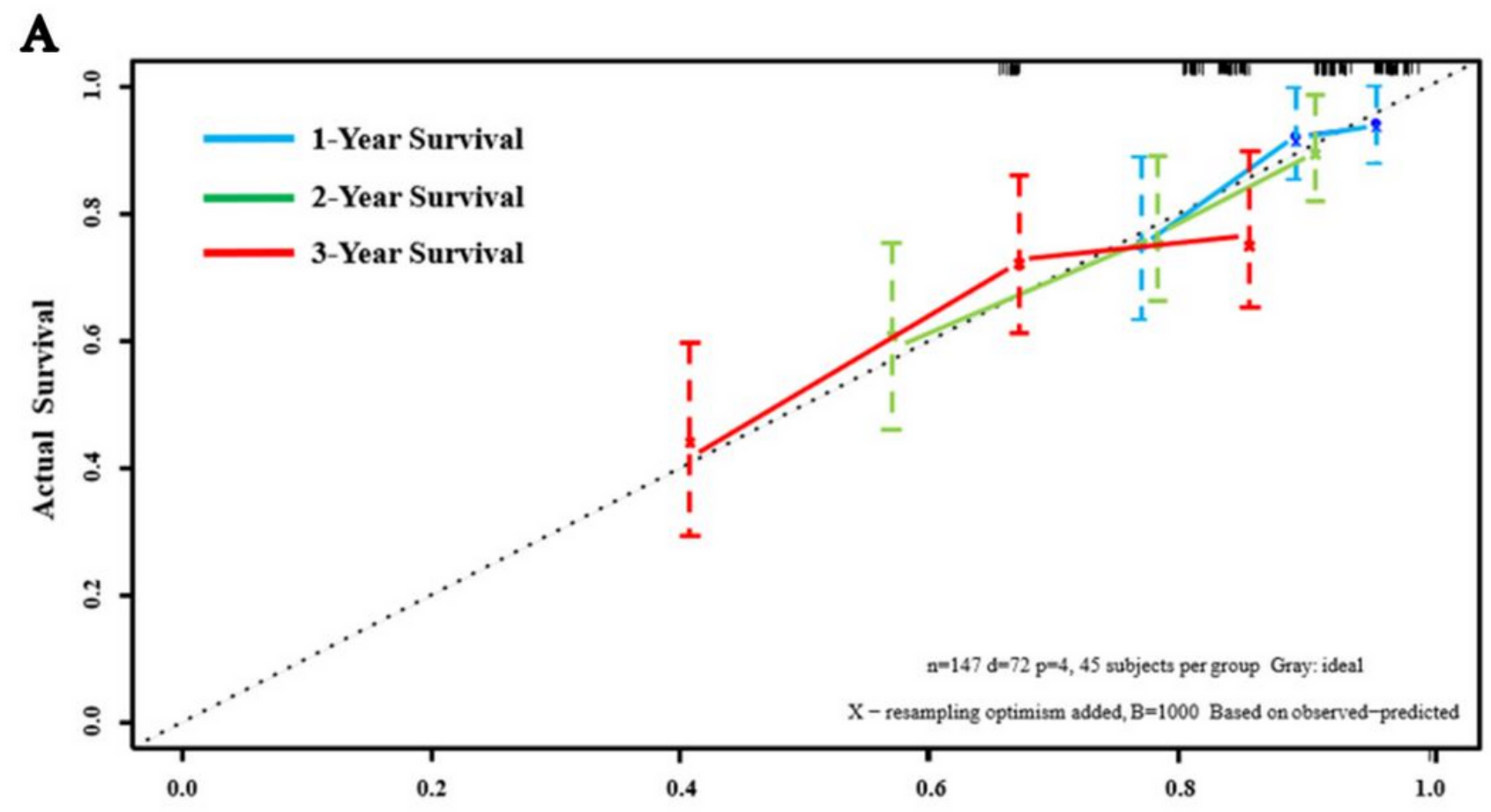

Nomogram-Predicted Probability of 1,2,3-Year Survival

B
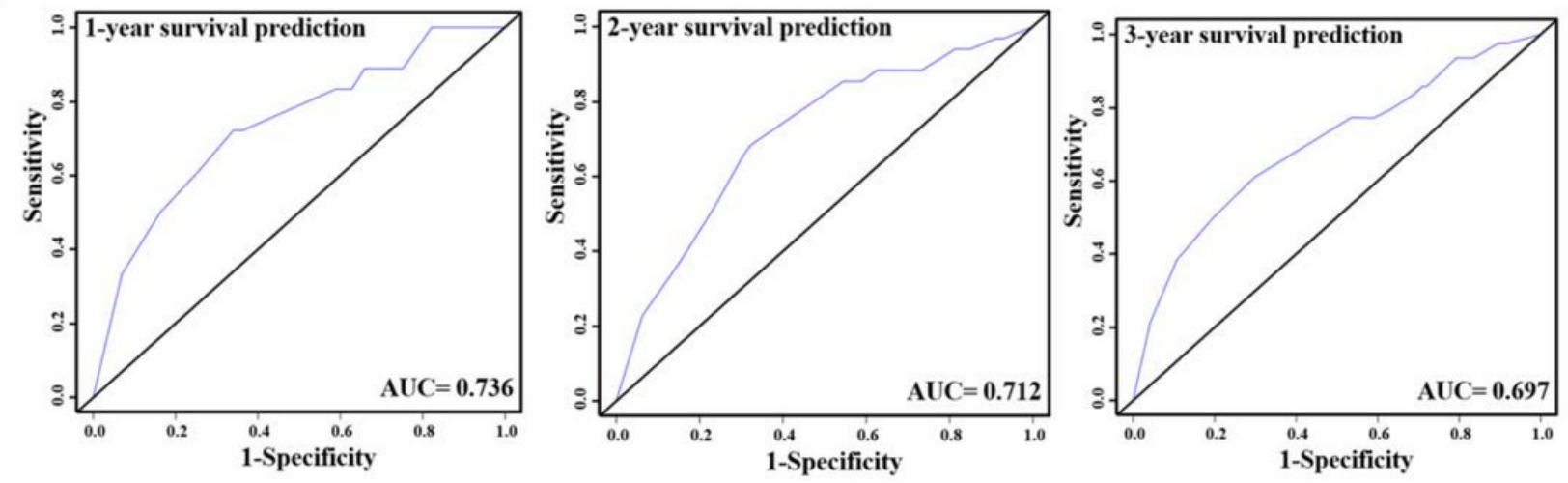

Figure 3

Calibration and Relative Operating Curve (ROC) of 1-, 2-, 3-year overall survival prediction. 3A. Calibration curve to validate nomogram model for survival and its C-index was $0.699(95 \% \mathrm{Cl}, 0.636-0.762)$. 3B. ROC curve of 1-, 2- and 3-year survival prediction, with area under the curve (AUC) $0.736,0.712$ and 0.697 . The dotted line in calibration curve represents the predictive value of the nomogram, while colorful lines represent actual value of 1-, 2-, 3-year OS rates, less discrepancy indicating more precise capability. 